\title{
Development of High-Speed Scan System for Atomic Resolution STEM
}

Yu Jimbo ${ }^{1}$, Ryo Ishikawa ${ }^{2}$, Mitsuhisa Terao ${ }^{3}$, Masashi Nishikawa ${ }^{3}$, Shigeyuki Morishita ${ }^{1}$, Masaki Mukai ${ }^{3}$, Hidetaka Sawada $^{3}$, Yuichi Ikuhara ${ }^{2}$ and Naoya Shibata ${ }^{4}$

${ }^{1}$ JEOL Ltd., Akishima, Tokyo, Japan, ${ }^{2}$ Institute of Engineering Innovation, University of Tokyo, Tokyo, Japan, ${ }^{3}$ JEOL Ltd., Akishima, Japan, ${ }^{4}$ Institute of Engineering Innovation, University of Tokyo, United States

Dynamic observation with transmission electron microscope (TEM) is expected to be a new tool to investigate atomic structure during reaction. Time resolved imaging with a fast acquisition is one of the important technologies for the dynamic observation. There are many studies using TEM, because we can perform fast data acquisition due to a high-speed CMOS camera. However, it is still difficult to distinguish element of atoms by phase contrast in TEM, and information about element species by image contrast is useful to understand single atom dynamics in atom cluster on carbon film, dopants in crystals, and so on. On the other hand, metallic atom detection on a carbon film by annular dark-field scanning transmission electron microscope (ADF-STEM) is reasonably feasible by z-contrast due to high signal-to-noise (S/N) ratio of single atom comparing the intensity of carbon atoms. Therefore, time-resolved observation with ADF-STEM is preferable to investigate dynamics of atomic motion in a cluster on a carbon film.

Higher order aberration corrector has been developed [1] to have improve spatial resolution, archiving the sub-angstrom resolution and the large probe current. The system can therefore reduce the dwell time of the scanning pixels owing to large probe current with reasonable $\mathrm{S} / \mathrm{N}$ ratio, so that we can increase the frame rate of ADF-STEM with keeping the sub-angstrom resolution.

We here develop a new high-speed scan system for JEM-ARM300F and JEM-ARM300F2 in collaboration with University of Tokyo. The target frame rate is $30 \mathrm{fps}$ at $512 \times 512$ pixels, where the fastest the dwell time is 83 ns per pixel. Detector and scan-coil have developed to improve the frame rate of STEM. Required response frequency of the detector is $6 \mathrm{MHz}$. Therefore, for the detector, we select the material of scintillator with a short lifetime, and optimize the electrical circuit for fast frequency. For the scan-coil, we use the low inductance coil to reduce the fly-back time with the optimized the circuit, resulting in that the system can reduce the fly-back time from $500 \mathrm{~ms}$ to $20 \mathrm{~ms}$, archiving the frame rate of $32 \mathrm{fps}$ in $512 \times 512$ pixels.

Figure 1 shows the ADF-STEM image of $\mathrm{SrTiO}_{3}$ obtained by the developed high-speed scan system. This image is superimposed 100 frames, where the accelerating voltage is $300 \mathrm{kV}$, the probe current is $44 \mathrm{pA}$, and the convergent half angle is $32 \mathrm{mrad}$. High- speed scan system can resolve atomic resolution images.

Figure 2(a) is the ADF-STEM images of a gold atom cluster obtained by the high-speed scan system, showing dynamics of gold atoms in the cluster. Each image of Fig.2(a) is superimposed by 2 frames, which are obtained with $32 \mathrm{fps}$, operating at $300 \mathrm{kV}$, the probe current is approximately $100 \mathrm{pA}$, and the dwell time is $83 \mathrm{~ns} /$ pix. Single atoms are visible in each image. Figure 2(b) shows the frame with interval of 31.25 msec. The atom indicated by white allow moved between these two frames. P. E. Batson et al.[2] reported that atomic movement was much faster than $200 \mathrm{msec}$. Our observation by developed fast scan system proved that the atomic movement is faster than $31.25 \mathrm{msec}$ [3]. 


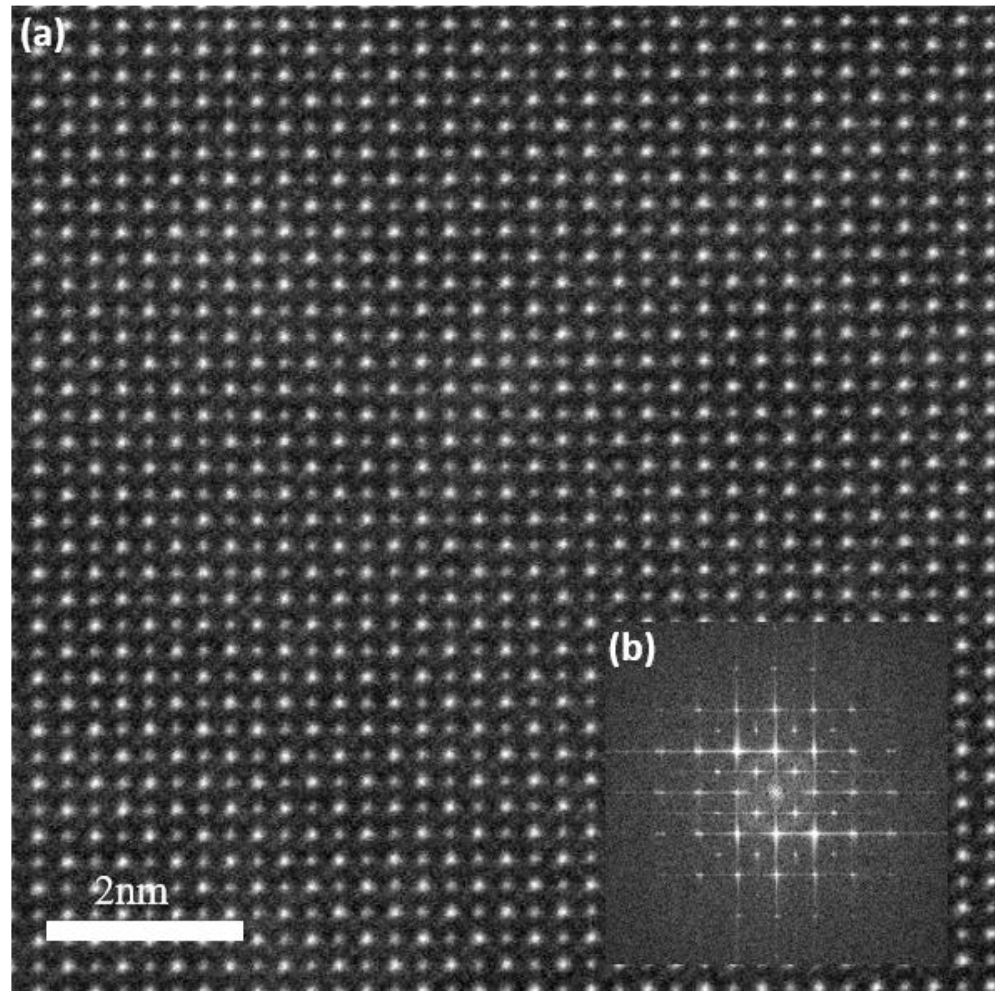

Figure 1. Figure 1. High-speed ADF STEM image of SrTiO3 superimposed 100 frames in (a) and the FFT in Fig.1(b), taken by the instrument of JEM-ARM300F2 equipped the higher order aberration corrector. The frame rate is $32 \mathrm{fps}$, the image pixel size is $512 \times 512$ pixels, the probe current is $44 \mathrm{pA}$ and the prove convergent angle is $32 \mathrm{mrad}$. Sub-angstrom resolution was achieved using high speed scan system. 

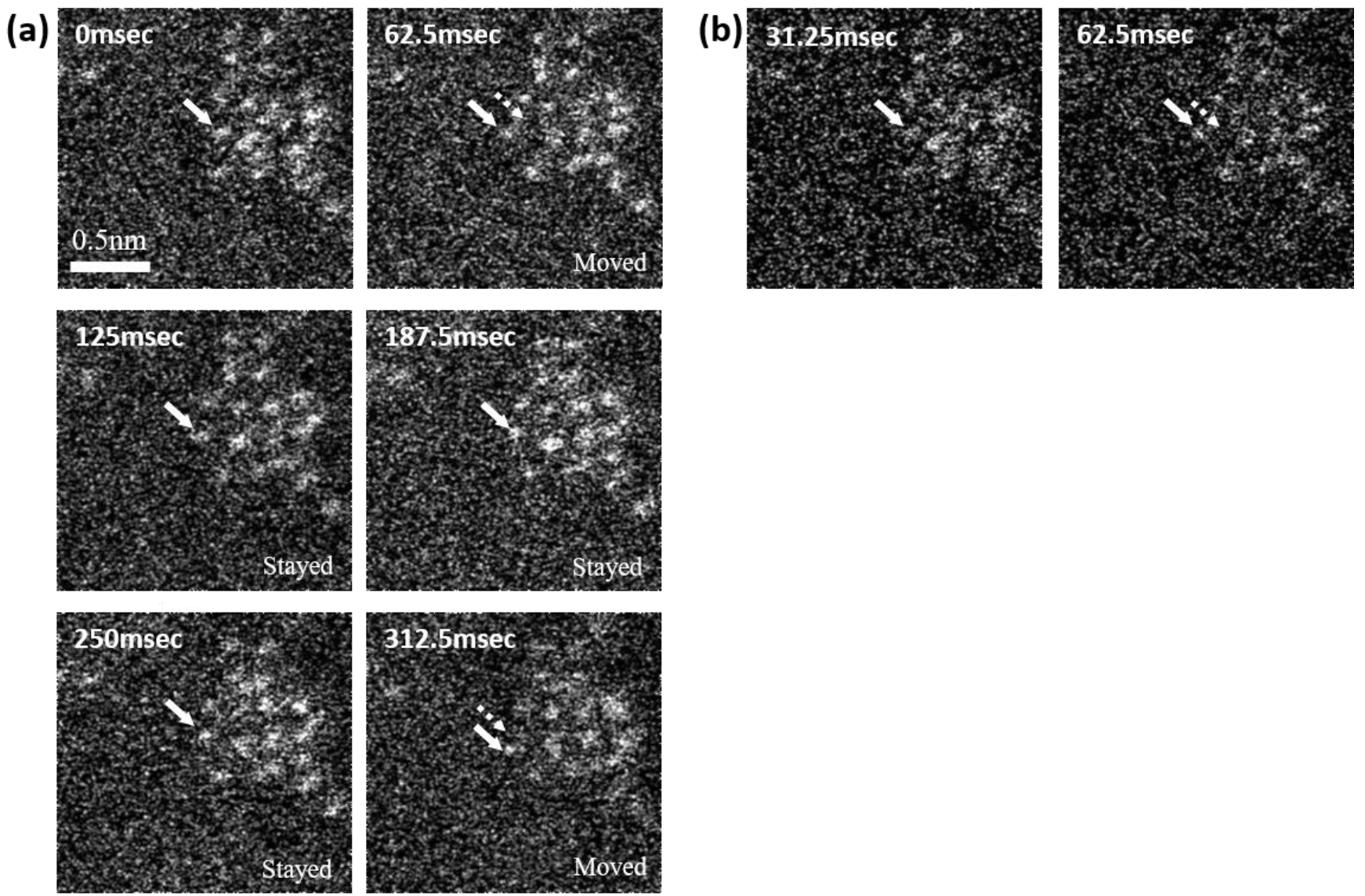

Figure 2. Figure 2. Motion of gold single atom is shown in (a). Each image is superimposed by 2 frames, and the frame speed is $31.25 \mathrm{msec}$ ( $32 \mathrm{fps}$ ). The dwell time is $83 \mathrm{~ns} / \mathrm{pix}$. The motion of gold atom could be tracked by high speed scan system. White arrows show the atom positions and the white dotted line indicate the original position. The time of atom movement is shown in Fig.2(b). These two images are 1 frame taken by $32 \mathrm{fps}$. The indicated atom moved between these frames. This proved the moving time of gold atom is shorter than $1 \mathrm{frame.}$

\section{References}

[1] S Morishita et al, Microscopy, 67 (2018) p.46.

[2] P.E.Batson et al, Microscopy and Microanalysis, 14(2008), p.89.

[3] R. Ishikawa et al, Microscopy 69240 (2020).

[4] A part of this work was supported by Grant-in-Aid for Specially

Promoted Research "Atom-by-atom imaging of ion dynamics in nano-structures

for materials innovation” (Grant No. JP17H06094) from JSPS. 